\title{
Structures in the cross section of double ionisation of helium by the impact of fast charged particles
}

\section{J. BERAKDAR}

\author{
Fakultät für Physik, Albert-Ludwigs-Universität, Hermann-Herder-Strasse 3, 79104 Freiburg i.Br., \\ Germany
}

\begin{abstract}
In this work the five-fold differential double-ionisation cross section of helium by the impact of fast charged particles has been investigated. The case is considered in which little momentum is transferred to the target so that a Born-type approximation in the projectile-target potential can be employed. A scaling formula for the cross section has been derived which relates cross sections resulting from the impact of projectiles of different masses and charges. Further, collision geometries have been found for which maxima and minima arise in the cross section and the associated ionisation mechanisms have been identified. Necessary kinematical conditions for the Bethe ridge in double ionisation have been worked out.
\end{abstract}

\section{Introduction}

Recent developments in coincidence measurements (Lahmam-Bennani et al 1989, Lahmam-Bennani et al 1992, Schwarzkopf et al 1993) have prompted an increase activity in the study of double ionisation. Theoretically, the double ionisation of helium by charged structureless particles is the simplest process leading to four unbound particles in the final channel. For high impact energy (compared to the bound-state energy) and small momentum being transferred to the target, a Borntype approximation in the projectile-target potential can be employed and the problem reduces to the description of the double-continuum state of the two ionized electrons in the field of the nucleus. Nevertheless, a complete solution for the latter problem is still outstanding. For the description of the double ionisation process by electron impact, the so-called (e,3e) process, several theoretical models have been proposed (Joulakian et al 1992, Joulakian and Dal Cappello 1993, Dal Cappello and Le Rouzo 1991, Tweed 1973, Smirnov et al 1978). These models operate within the Born approximation. The three-body state consisting of the nucleus and the two-ionized electrons is then described by some approximate wavefunction. In the present work, we consider the double ionisation of helium by the impact of arbitrary charged structureless projectiles and we obtain a scaling formula for the cross section. Hence, it is sufficient to confine the consideration to (e,3e) reaction since other cases can then be obtained by scaling. It is the aim of this paper, within the Born approximation and else from quite general point of view, to provide kinematical conditions under which maxima and minima appear in the cross section and to identify the associated mechanisms leading to these structures. In particular, the effects of initial- and final-state correlations for the target electrons have been 
considered. Further, the symmetry properties of the cross section have been pointed out which considerably restrict the kinematical region in which the cross section have to be investigated. This is of vital importance for experiments since the cross section depends on large number of independent variables.

The most complete information about the $(\mathrm{e}, 3 \mathrm{e})$ collision process is obtained by measuring the fivefold differential cross section, hereafter FDCS, where energy- and angle-resolved detection of the two ionized electrons is performed in coincidence with the scattering solid angle of the projectile. Such experiments have been carried out using argon and krypton targets (Lahmam-Bennani et al 1989, Lahmam-Bennani et al 1992, see Lahmam-Bennani 1991 for a comprehensive review). Due to technical difficulties, however, the FDCS for helium target has not yet been measured. Detecting only two of the three electrons present in the final state, we end up with the (e,3-1e) experiments (LahmamBennani et al 1991, Duguet and Lahmam-Bennani 1992). Atomic units are used throughout.

\section{Theoretical treatment}

We study the case where a fast (with respect to the Bohr velocity of the bound electrons) incoming projectile of mass $M_{P}$ and charge $Z_{P}$ double ionizes the helium atom in its singlet ground state. In this work we focus on the collision geometry where only a small amount of momentum is transferred from the projectile to the target atom. This geometry yields the largest cross section and hence it is of major interest. The two-electron bound-free transition is then described within the first Born approximation (FBA) with respect to the projectile-target potential. The projectile is then treated before and after the collision as a free particle. In the centre-of-mass system, this four-body problem can be described by a set of Jacobi coordinates, e.g. $\left(\mathbf{r}_{a}, \mathbf{r}_{b}, \mathbf{r}_{0}\right)$ or $\left(\mathbf{R}, \mathbf{r}, \mathbf{r}_{0}\right)$ (see figure 1 for explanation of notations). The initial state is then described by the product wavefunction

$$
\Phi_{i}\left(\mathbf{r}_{0}, \mathbf{r}_{a}, \mathbf{r}_{b}\right)=(2 \pi)^{-2 / 3} \exp \left(\mathbf{k}_{i} \mathbf{r}_{0}\right) \varphi\left(\mathbf{r}_{a}, \mathbf{r}_{b}\right)
$$

where $\mathbf{k}_{i}$ is the momentum of the incident projectile with respect to the centre of mass of the helium atom. The two-electron bound-state wavefunction for the $\mathrm{He}\left({ }^{1} S^{e}\right)$ ground state is denoted by $\varphi\left(\mathbf{r}_{a}, \mathbf{r}_{b}\right)$. The momentum transfer to the target is given by $\mathbf{q}=\mathbf{k}_{i}-\mathbf{k}_{0}$ where $\mathbf{k}_{0}$ is the momentum of the scattered projectile. For the case of electron impact, exchange between the projectile electron and the two target electrons can be disregarded because at small values of momentum transfer and high impact energy the scattered electron is much faster than the ejected electrons. Assume now that the two atomic electrons labeled by indices $a$ and $b$ are ejected into differential solid angles $d \Omega_{a}$ and $d \Omega_{b}$, and the projectile is scattered into the solid angle $d \Omega_{0}$. Within the FBA, integration over the projectile coordinate $r_{0}$ can be immediately carried out (Bethe 1930, Inokuti 1973, Inokuti et al 1978). The FDCS being differential in the directions $\Omega_{a}, \Omega_{b}, \Omega_{0}$ and in energies of the two secondary electrons $E_{a}$ and $E_{b}$ is then given by

$$
\frac{d^{5} \sigma}{d \Omega_{a} d \Omega_{b} d \Omega_{0} d E_{a} d E_{b}}=(2 \pi)^{4} \mu_{a} \mu_{b} \mu_{P T}^{2} Z_{P}^{2} \frac{k_{a} k_{b} k_{0}}{k_{i}}\left(\frac{4 \pi}{q^{2}}\right)^{2}\left|T_{f i}\right|^{2} .
$$

where the reduced masses $\mu_{a}, \mu_{b}$ and $\mu_{P T}$ are given by

$$
\mu_{a}=\frac{M_{T}}{M_{T}+1}, \quad \mu_{b}=\frac{M_{T}+1}{M_{T}+2}, \text { and } \mu_{P T}=\frac{M_{P}\left(M_{T}+2\right)}{M_{P}+M_{T}+2} .
$$




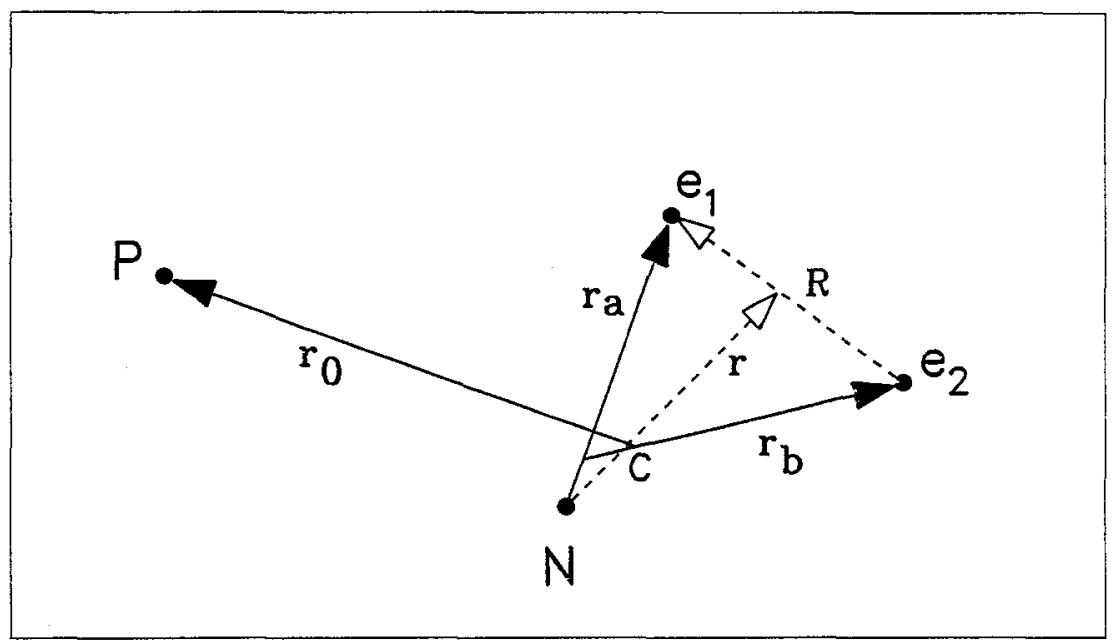

Figure 1: The Jacobi coordinates for the four-body system. The nucleus-electron (N-e1) relative coordinate is denoted by $\mathrm{r}_{a}$. The relative coordinate of the other target electron $\left(\mathbf{e}_{2}\right)$ with respect to the subsystem $\left(\mathbf{N}-\mathbf{e}_{1}\right)$ is labeled by $\mathbf{r}_{b}$. The position of the projectile $(\mathrm{P})$ relatively to the centre of mass of the helium atom (C) is denoted by $\mathbf{r}_{0}$. An alternative set of Jacobi coordinates is $\left(\mathbf{r}, \mathbf{R}, \mathbf{r}_{0}\right)$ where $\mathbf{R}$ is the relative coordinate of the $\left(\mathbf{e}_{1}, \mathbf{e}_{2}\right)$ subsystem and $\mathbf{r}$ denotes the relative coordinate of the center of mass of this subsystem with respect to the nucleus.

Here $M_{T}$ is the mass of the nucleus. The two-electron transition matrix element $T_{f i}$ is defined by

$$
T_{f i}=<\psi_{\mathbf{k}_{a}, \mathbf{k}_{b}}^{-}\left(\mathbf{r}_{a}, \mathbf{r}_{b}\right)|W| \varphi\left(\mathbf{r}_{a}, \mathbf{r}_{b}\right)>
$$

where the operator $W$ is given by (terms of the order $q / M_{T}$ have been neglected).

$$
W=Z_{T}-\exp \left(i \mathbf{q} \mathbf{r}_{a}\right)-\exp \left(i \mathbf{q} \mathbf{r}_{b}\right)
$$

and $Z_{T}=2$ is the $H e$ nucleat charge. Thus, the $T$-matrix element (4) consists of three transition amplitudes,

$$
T_{f i}=T_{T}-T_{a}-T_{b}
$$

where $T_{T}, T_{a}, T_{b}$ stem from the interaction of the impinging projectile with the target nucleus and the two target electrons, respectively,

$$
\begin{aligned}
T_{T} & =\left\langle\psi_{\mathbf{k}_{a}, \mathbf{k}_{b}}^{-}\left(\mathbf{r}_{a}, \mathbf{r}_{b}\right)\left|Z_{T}\right| \varphi\left(\mathbf{r}_{a}, \mathbf{r}_{b}\right)\right\rangle \\
T_{a} & =\left\langle\psi_{\mathbf{k}_{a}, \mathbf{k}_{b}}^{-}\left(\mathbf{r}_{a}, \mathbf{r}_{b}\right)\left|\exp \left(i \mathbf{q} \cdot \mathbf{r}_{a}\right)\right| \varphi\left(\mathbf{r}_{a}, \mathbf{r}_{b}\right)\right\rangle \\
T_{b} & =\left\langle\psi_{\mathbf{k}_{a}, \mathbf{k}_{b}}^{-}\left(\mathbf{r}_{a}, \mathbf{r}_{b}\right)\left|\exp \left(i \mathbf{q} \cdot \mathbf{r}_{b}\right)\right| \varphi\left(\mathbf{r}_{a}, \mathbf{r}_{b}\right)\right\rangle
\end{aligned}
$$

The momentum and energy conservation are expressed through the equations,

$$
\mathbf{q}=\mathrm{k}_{a}+\mathbf{k}_{b}+\mathbf{k}_{i o n}
$$

and

$$
E_{i}-\epsilon=E_{0}+E_{a}+E_{b}+E_{i o n}
$$


where $\mathbf{k}_{i o n}$ is the recoil momentum of the nucleus and $E_{i}=k_{i}^{2} / 2 \mu_{P T}, E_{0}=k_{0}^{2} / 2 \mu_{P T}, E_{\alpha}=k_{b}^{2} / 2 \mu_{a}$, $E_{b}=k_{b}^{2} / 2 \mu_{b}$ and $E_{i o n}=k_{i o n}^{2} / 2 M_{T}$ are the energies of the incoming, the scattered particle, the two ionized electrons and the recoiling nucleus, respectively, and $\epsilon \approx 2.9037$ is the (positive) binding energy of both target electrons.

The wavefunction $\psi_{\mathbf{k}_{a}, \mathbf{k}_{b}}^{-}\left(\mathbf{r}_{a}, \mathbf{r}_{b}\right)$ in eq.(3) should be an exact eigenfunction for the helium atom in the double continuum with boundary conditions describing two outgoing electrons, i.e. to the order $1 / M_{T}$

$$
\left(-\frac{1}{2} \nabla_{\mathbf{r}_{a}}^{2}-\frac{1}{2} \nabla_{\mathbf{r}_{b}}^{2}-\frac{Z_{T}}{r_{a}}-\frac{Z_{T}}{r_{b}}+\frac{1}{r_{a b}}-E_{a}-E_{b}\right) \psi_{\mathbf{k}_{a}, \mathbf{k}_{b}}^{-}\left(\mathbf{r}_{a,}, \mathbf{r}_{b}\right)=0
$$

If the wavefunctions $\psi_{\mathbf{k}_{a}, \mathbf{k}_{b}}^{-}\left(\mathbf{r}_{a}, \mathbf{r}_{b}\right)$ and $\varphi\left(\mathbf{r}_{a}, \mathbf{r}_{b}\right)$ are eigenfunctions of the same Hamiltonian (12), the term $T_{T}$ in equation (6) vanishes due to orthogonality of different target states. In general, however, since no exact solution of the three-body problem (12) exists there is a finite overlap due to nonorthogonality of approximate initial and final-state wavefunctions and the term $T_{T}$ in eq.(6) provides a finite contribution to the $T$-matrix element. Nevertheless, initial- and final-state wavefunctions can be made orthogonal according to the Schmidt prescription by defining a final-state wavefunction as,

$$
\bar{\psi}_{\mathbf{k}_{a}, \mathbf{k}_{b}}^{-}\left(\mathbf{r}_{a}, \mathbf{r}_{b}\right)=\psi_{\mathbf{k}_{a}, \mathbf{k}_{b}}^{-}\left(\mathbf{r}_{a}, \mathbf{r}_{b}\right)-\frac{T_{T}^{*}}{Z_{T}} \varphi\left(\mathbf{r}_{a}, \mathbf{r}_{b}\right)
$$

The $T$-matrix element calculated with (13) instead of $\psi_{\mathbf{k}_{a}, \mathbf{k}_{b}}\left(\mathbf{r}_{a}, \mathbf{r}_{b}\right)$ has then no contribution from $T_{T}$ because of orthogonality $\left\langle\bar{\psi}_{\mathbf{k}_{a}, \mathbf{k}_{b}}^{-}\left(\mathbf{r}_{a}, \mathbf{r}_{b}\right)\right| \varphi\left(\mathbf{r}_{a}, \mathbf{r}_{b}\right)>=0$. Though the $T$-matrix calculated using (13) or alternatively $\psi_{\mathbf{k}_{a}, \mathbf{k}_{b}}\left(\mathbf{r}_{a}, \mathbf{r}_{b}\right)$ approaches, in both cases, correctly the optical limit (Berakdar and Klar 1993) for vanishing values of momentum transfer, results obtained using (13) and $\psi_{\mathbf{k}_{a}, \mathbf{k}_{b}}\left(\mathrm{r}_{a}, \mathrm{r}_{b}\right)$ are in general not identical, increasingly so for increasing violation of orthogonality.

Thus, we stress that there is no guarantee that results obtained using (13) will be more accurate than those obtained using $\psi_{\mathbf{k}_{a}, \mathbf{k}_{b}}^{-}\left(\mathbf{r}_{a}, \mathbf{r}_{b}\right)$ since the wavefunction (13) contains the physically spurious term $T_{T}$ which is essentially due to a poor description of the three-body state and thus it depends on the approximate model used. Therefore, structures in the FDCS which arise primary from $T_{T}$ are physically doubtful.

Finally, we come to symmetries. Since the $H e$ ground state is a singlet, its spatial wavefunction is even with respect to exchange. Since also the transition operator (5) is symmetric in configuration space, the final state is symmetric, i.e. the relation

$$
\psi_{\mathbf{k}_{a}, \mathbf{k}_{b}}^{-}\left(\mathbf{r}_{a}, \mathbf{r}_{b}\right)=\psi_{\mathbf{k}_{a}, \mathbf{k}_{b}}^{-}\left(\mathbf{r}_{b}, \mathbf{r}_{a}\right)
$$

holds. The two-electron final state wave function given by (12) has to describe two outgoing electrons. Its asymptotic behaviour, therefore, is that of Coulomb modified plane waves (see Rosenberg 1973). This Redmond asymptotic, and therefore the whole solution, has the following symmetry properties in the phase space:

$$
\begin{aligned}
& \psi_{\mathbf{k}_{a}, \mathbf{k}_{b}}^{-}\left(\mathbf{r}_{a}, \mathbf{r}_{b}\right)=\psi_{\mathbf{k}_{b}, \mathbf{k}_{a}}^{-}\left(\mathbf{r}_{b}, \mathbf{r}_{a}\right) \\
& \psi_{\mathbf{k}_{a}, \mathbf{k}_{b}}\left(\mathbf{r}_{a}, \mathbf{r}_{b}\right)=\psi_{-\mathbf{k}_{a},-\mathbf{k}_{b}}^{-}\left(-\mathbf{r}_{a},-\mathbf{r}_{b}\right)
\end{aligned}
$$

Eqs. (14) and (15) may also be combined to give

$$
\psi_{\mathbf{k}_{a}, \mathbf{k}_{b}}^{-}\left(\mathbf{r}_{a}, \mathbf{r}_{b}\right)=\psi_{\mathbf{k}_{b}, \mathbf{k}_{a}}^{-}\left(\mathbf{r}_{a}, \mathbf{r}_{b}\right)
$$




\subsection{The scaling law}

The scope of this section is to derive a formula which will enable us to compare cross sections resulting from the impact of projectiles of different masses and charges. To this end we choose the electron as a reference projectile. The initial and final momenta of a projectile with mass $M_{P}$ and charge $Z_{P}$ are then chosen such that the momentum transfer vector $q$ for this case is equal to that resulting from electron impact. Additionally, we assume that arbitrary projectiles have the same initial velocity as the projectile electron and also that the target electrons ionized by the impact of different projectiles have the same final momenta $\mathbf{k}_{a}, \mathbf{k}_{b}$.

For a given incident energy $E_{0}$ of the electron we obtain, under these conditions, a projectile of mass $M_{P}$ will have the incident energy $E_{i}$

$$
E_{i}=\mu_{P T} E_{0}
$$

where the reduced mass $\mu_{P T}$ is given by (3). Further, assuming the projectile electron has incident momentum $k_{0}$, the scattering angle of the electron $\theta$ is then related to the scattering angle $\Theta$ of an arbitrary projectile of mass $M_{P}$ through the relation

$$
\theta=\sin ^{-1}\left(\frac{k_{0}\left(\mu_{P T}-1\right) \sin (\Theta)}{\left(2 \epsilon+k_{0}^{2}-k_{a}^{2}-k_{b}^{2}\right)^{1 / 2}}\right)+\Theta .
$$

For this choice of kinematics we define a scaled cross section (FDCS*) as

$$
F D C S^{*}=F D C S / C \quad \text { where } \quad C=Z_{P}^{2} \mu_{P T}^{2} k_{0} / k_{i} .
$$

Within the present frame of description the FDCS* as function of $\mathbf{k}_{a}$ or/and $\mathbf{k}_{b}$ is then identical for projectiles of arbitrary mass and charge.

\subsection{Maxima and minima in the FDCS}

According to the scaling formula (20), it is sufficient to confine the considerations of the FDCS to the case of electron impact. The FDCS resulting by the impact of other projectiles are then obtained by scaling. The following analysis is based on the Born approximation for the four-body problem. The three-body wavefunctions $\psi_{\mathbf{k}_{a}, \mathbf{k}_{b}}^{-}\left(\mathbf{r}_{a}, \mathbf{r}_{b}\right)$ and $\varphi\left(\mathbf{r}_{a}, \mathbf{r}_{b}\right)$, however, are assumed to be exact. Let us consider the case $\mathbf{k}_{a}=-\mathbf{k}_{b}$ for which the exact wavefunction $\psi_{\mathbf{k}_{a}, \mathbf{k}_{b}}^{-}\left(\mathbf{r}_{a}, \mathbf{r}_{b}\right.$ ) has even parity (Berakdar and Klar 1993). For this case, the first non vanishing term in the $T$-matrix eq. (4) is proportional to $q^{2}$ and only terms proportional to $q^{2 n}$ and $n=$ integer appear in the $T$-matrix. Therefore, for fixed $\mathbf{k}_{b}$ and small fixed $q$, a minimum occurs in the FDCS as function of $\mathbf{k}_{a}$ at $\mathbf{k}_{a}=-\mathbf{k}_{b}$. For small $q$ this minimum appears also in the momemtum distribution of electron " $b$ " at $\mathbf{k}_{b}=-\mathbf{k}_{a}$ where $\mathbf{k}_{a}$ and $q$ are fixed. Further, this minimum becomes a zero for dipole transitions (Maulbetsch and Briggs 1993, Huetz et al 1991, Teng and Shakeshaft 1993).

In the case of very high impact energies and not too small momentum transfer the position vectors of the ionized electrons $\mathbf{r}_{a}, \mathbf{r}_{b}$ are almost parallel to the directions $\mathbf{k}_{a}, \mathbf{k}_{b}$ respectively. In this situation and for fixed $q$, a zero point in the angular distribution of ejected electrons is observed whenever $\hat{\mathbf{k}}_{a} \perp \mathbf{q}$ and $\hat{\mathbf{k}}_{b} \perp \mathbf{q}$. In general, this zero point becomes a minimum in the above mentioned cross section since the probability for a dipole transition vanishes (Maulbetsch and Briggs 1993,Huetz et al 1991, Teng and Shakeshaft 1993) and the first leading term in the $T$-matrix for (e,3e) is given by $q^{2}$. For the case $\mathbf{k}_{a}=\mathbf{k}_{b}$, the electron-electron repulsion leads to vanishing FDCS. 
The kinematical origins of these minima have been exposed by Berakdar and Klar (1993). Maxima in the FDCS as function of $\mathbf{k}_{a}$ and/or $\mathbf{k}_{b}$ are expected to occur for kinematical conditions under which the electrons are directly ionized and the momentum transfer to the nucleus is minimized. This situation resembles the well known Bethe ridge in (e,2e) reactions (Lahmam-Benanni 1991). In order to find out these conditions we employ the momenta

$$
\begin{aligned}
\mathbf{K} & =\frac{1}{2}\left(\mathbf{k}_{a}+\mathbf{k}_{b}\right) \\
\mathbf{L} & =\frac{1}{2}\left(\mathbf{k}_{a}-\mathbf{k}_{b}\right) .
\end{aligned}
$$

These momenta $\mathbf{K}$ and $\mathbf{L}$ are conjugate to the Jacobi coordinates $\mathbf{r}, \mathbf{R}$ (see figure 1). Now for fixed incident energy $E_{i}$ and fixed momentum transfer $\mathbf{q}$ we demand $\mathbf{k}_{i o n}=0$. The momentum and energy conservation laws eq. $(10,11)$ lead then to the equations

$$
\begin{aligned}
\mathbf{K} & =\frac{1}{2} \mathbf{q} \\
L^{2} & =E_{q}-K^{2}
\end{aligned}
$$

where $E_{q}$ is the energy transferred to the target, i.e.

$$
E_{q}=\left(k_{a}^{2}+k_{b}^{2}\right) / 2=E_{a}+E_{b} .
$$

From $(21,22)$ we deduce further

$$
\begin{aligned}
\mathbf{k}_{a} \cdot \mathbf{k}_{b} & =\frac{q^{2}}{2}-E_{q} \\
\hat{\mathbf{q}} \cdot \hat{\mathbf{k}}_{a} & =\frac{q^{2}-2\left(E_{b}-E_{a}\right)}{2 q k_{a}} \\
\hat{\mathbf{q}} \cdot \hat{\mathbf{k}}_{b} & =\frac{q^{2}-2\left(E_{a}-E_{b}\right)}{2 q k_{b}} \\
\hat{\mathbf{q}} \cdot \hat{\mathbf{L}} & =\frac{2\left(E_{a}-E_{b}\right)}{q \sqrt{4 E_{q}-q^{2}}}
\end{aligned}
$$

From equations (22) it is clear that the condition $\mathbf{k}_{i o n}=\mathbf{0}$ fixes the momentum $\mathbf{K}$ and the magnitude of the momentum $\mathbf{L}$. However, the relations (22) still allow for varying $\hat{\mathbf{L}}$, i.e. the Bethe conditions (22) are fulfilled on a two dimensional sphere spanned by $\hat{\mathbf{L}}$ in the six dimensional momentum space $\mathbf{K} \otimes \mathbf{L}$. In the following we will denote this sphere by the Bethe sphere.

Geometrically the Bethe sphere can by illustrated in a simple way (figure 2a). The Bethe sphere $\mathcal{S}$ (figure 2a) has the radius $L / 2$. The center of $\mathcal{S}$ is the crossing point of $\mathbf{L}$ and $\mathbf{K}$ which is also the midpoint of $\mathbf{L}$ and $\mathbf{K}$. The cylindrical symmetry of the FDCS as function of $\hat{\mathbf{L}}$ around $\mathbf{K}$ for momenta lying on $\mathcal{S}$ is obvious from figure (2a). Further, figure (2a) elucidate the symmetry of the FDCS around $\hat{\mathbf{q}} \cdot \hat{\mathbf{L}}=\mathbf{0}$ due to the indistinguishibilty of the two electrons. Varying the polar angle $\theta_{L}=\cos ^{-1}(\hat{\mathbf{q}} \cdot \hat{\mathbf{L}})$ energies and polar angles of the two ejected electrons change according to (24). However, as can be seen from figures $(2 \mathrm{a}, 2 \mathrm{~b})$ the momenta of the ejected electrons $k_{b}$ and $k_{a}$ are restricted to the interval $[L-K, L+K]$.

Now whenever the Bethe sphere $\mathcal{S}$ is approached we expect an increase in the FDCS. On $\mathcal{S}$ itself, however, the FDCS is not constant and the structures arising in the FDCS in this case are directly traced to the electron-electron correlations because only single-particle operators are present in the 


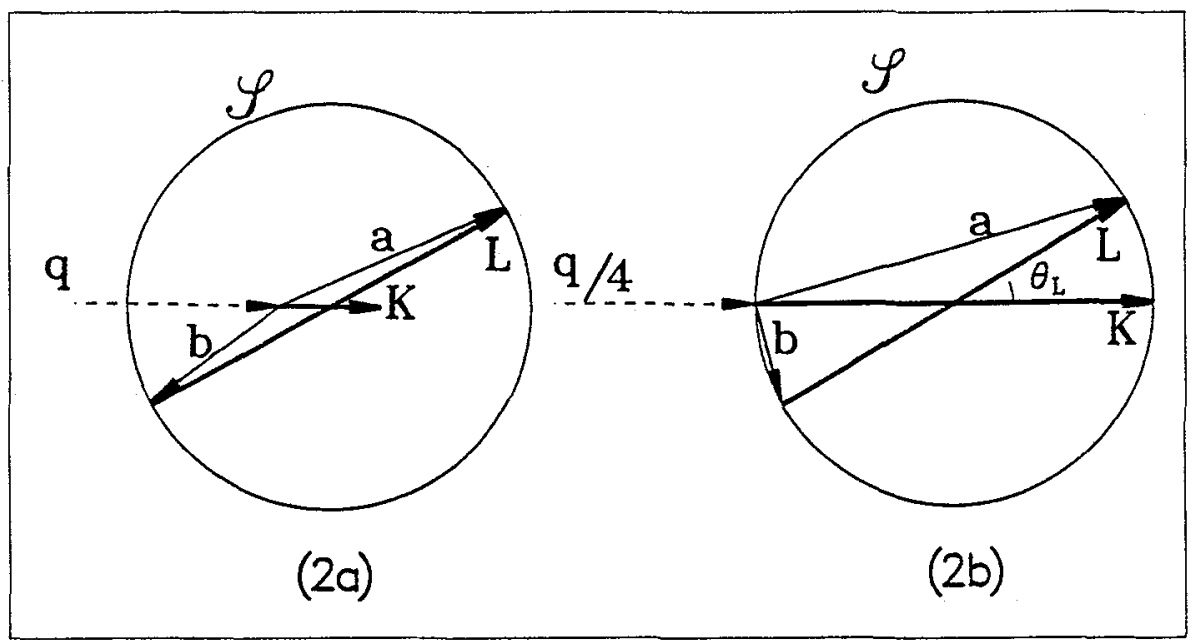

Figure 2: The Bethe conditions (22) are fulfilled on the two-dimensional Bethe sphere $\mathcal{S}$. The momenta $\mathrm{k}_{a} / 2$ and $\mathbf{k}_{b} / 2$ are labeled by $\mathbf{a}$ and $\mathrm{b}$ respectively. The two limiting cases of $\mathcal{S}$ are shown. For the case $L \gg K(2 \mathrm{a})$ the electron ejection energies $E_{a}$ and $E_{b}$ vary within a very limited energy region (compare eqs. 24 ). In the case $(2 \mathrm{~b}) L=K$ and hence the momenta $\mathrm{k}_{a} / 2$ and $\mathrm{k}_{b} / 2$ are always perpendicular when varied on $\mathcal{S}$.

perturbation $W$ (eq.5), i.e. the other electron is ionized by means of correlation with the first ejected electron. Therefore, we consinder the FDCS where the momenta $\mathrm{k}_{a}$ and $\mathrm{k}_{b}$ lie on $\mathcal{S}$ to identify collision geometries for which the FDCS is largest. From figures ( $2 a$ and $2 b$ ) it is clear that there are two limiting cases for the structure of $\mathcal{S}$. In the first case (figure $2 \mathrm{a}$ ), $L \gg K$, i.e. $2 E_{q} / q^{2} \gg 1$ (compare eqs.22). The momenta $\mathbf{k}_{a}$ and $\mathbf{k}_{b}$ are then almost equal in magnitude but have nearly opposite directions (the case $k_{a}=-k_{b}$ on $\mathcal{S}$ is excluded since $\mathrm{q}=0$ ). With varying $\theta_{L}$ energies $E_{a}$ and $E_{b}$ of the ejected electrons are restricted to a very limited energy region $[L-K, K+L]$ since $L \gg K$. In case of figure $2 \mathrm{a}$ the incoming projectile ionizes the two electrons simultaneously. Therefore, the FDCS as function of $\theta_{L}$ will show a maximum at $\theta_{L}=0$ (and $\pi$ ) in which case $L$ lies in the $q$-direction. Since the projectile does not simultaneously interact with the two target electron , (see eq.5) this transition must occur via a coherent superposition of the two transition amplitudes $T_{a}$ and $T_{b}$ (eq.4).

The second special case is $E_{q}=q^{2} / 2$. From eqs. (22 and 24) we deduce then $L=K$ and $\mathbf{k}_{a} \perp \mathbf{k}_{b}$ (see figure $2 b$ ). Now for a given q, i.e. $\mathbf{K}$ fixed, we consider the FDCS as function of $\theta_{L}$. At $\theta_{L}=0$ electron " $a$ " escapes in the q-direction carrying almost the whole momentum transferred to the target whereas the slow electron " $b$ " escapes in the opposite direction. This results in an increased FDCS at $\theta_{L}=0$. The mechanism leading to this structure is a shakeoff process (Carlson and Krause 1965, Carlson 1967) where the fast electron " $a$ " is ejected in the direction q after a hard collision with the projectile. Electron " $b$ " is then shaken off by the sudden change of the effective nuclear charge. Therefore the main contribution to the $T$-matrix element, eq.(4), originates from the transition amplitude $T_{a}$. The angular distribution of the slow electron " $b$ " then reflects directly 
the angular structure of the bound state. For $\theta_{L}=\pi / 2$ the momenta $k_{a}$ and $k_{b}$ are equal. In this case we expect a peak in the FDCS due to a sequentail knock-out collision (Byron and Joachain 1967, McGuire 1982, Ishihara et al 1991). In the present case this process can be intepreted as follows. One of the two electrons, say electron " $a$ ", is ejected after a hard collision with the projectile into the direction $\mathbf{q}$. Then it undergoes a second hard collision with electron " $b$ " and escapes into a direction perpendicular to $\mathbf{k}_{b}$. As can be seen from eq.(4) this transition must occur due to final-state correlations between the two target electrons. The transition amplitudes $T_{a}$ and $T_{b}$ are equal in this case (see figures $2 \mathrm{a}-2 \mathrm{~b}$ ). At $\theta_{L}=\pi$ electron " $b$ " is knocked out by a hard collision with the projectile whereas electron " $a$ " is shaken off. Therefore, the transition amplitude $T_{b}$ (eq.4) provides in this case the main contribution to the $T$-matrix element. The above findings have been confirmed by numerical calculations (Berakdar and Klar 1993).

\section{Conclusions}

In this work the FDCS for charged particle impact of helium has been studied. Cross sections resulting from the impact of different projectiles have been related by scaling. Minima and maxima in the FDCS have been investigated and the mechanisms leading to these stucutres have been exposed. Necessary kinematical conditions for the Bethe ridge have been derived. The symmetry properties of the crosss section as well as of the wavefunctions have been pointed out.

\section{Acknowledgement}

This work has been supported by the Deutsche Forschungsgemeischaft under contract SFB 276 .

\section{References}

Berakdar J and Klar H 1993 to be published

Bethe H 1930 Ann. Phys., Lpz. 5325

Byron F W, Joachain C J 1967 Phys. Rev. 1641 J. Phys. B: At. Mol. Phys. 183203

Carlson T A 1967 Phys. Rev. 156142

Carlson T A and Krause M O 1965 Phys. Rev. 1401057

Dal Cappello C and Le Rouzo H 1991 Phys, Rev, A 431395

Duguet A and Lahmam-Bennani A 1992 Z. Phys. D 23383

Huetz A, Selles P, Waymel D and Mazeau J 1991 J. Phys. B: At. Mol. Phys. 241917

Ishihara T, Heno K and McGuire J H 1991 Phys. Rev. A 44 R6980

Inokuti M 1971 Rev. Mod. Phys. 43297

Inokuti M, Itikawa Y and Turner J E 1978 Rev. Mod. Phys. 5023

Joulakian B, Dal Cappello C and Brauner M 1992 J. Phys. B: At. Mol. Phys. 252863

Joulakian B and Dal Cappello C 1992 Phys. Rev. A 473788

Le Rouzo H and Dal Cappello C 1991 Phys. Rev. A 43318 
Lahmam-Bennani A 1991 J. Phys. B: At. Mol. Phys. 242401

Lahmam-Bennani A, C. Dupŕe and Duguet A 1989 Phys. Rev. Lett. 631582

Lahmam-Bennani A, Duguet A, Grisogono A M and Lecas M 1992 J. Phys. B: At. Mol. Phys. 252873

Maulbetsch F and Briggs J S 1993 J. Phys. B: At. Mol. Phys. 261679

McGuire J H 1982 Phys. Rev. Lett. 491153

Rosenberg L 1973 Phys. Rev. D 81833

Schwarzkopf O, Krässing B, Elmiger J and V Schmidt 1993 Phys. Rev. Lett. 703008

Smirnov Yu F, Pavlitchenkov A V, Levin V G and Neudatchin G 1978 J. Phys. B: At. Mol. Phys. 1120

Teng $\mathrm{Z}$ and Shakeshaft R 1993 Phys. Rev. A (part A) 47 R3487

Tweed R J 1973 J. Phys. B: At. Mol. Phys. 6270 